\title{
Chronic pain after surgery: pathophysiology, risk factors and prevention
}

\author{
Danielle Reddi, ${ }^{1}$ Natasha Curran ${ }^{2}$
}

\begin{abstract}
${ }^{1}$ Centre for Anaesthesia, University College London Hospital, London, UK ${ }^{2}$ Pain Management Centre, National Hospital for Neurology and Neurosurgery, London, UK
\end{abstract}

\section{Correspondence to} Dr Danielle Reddi, Centre for Anaesthesia, University College London Hospital, London NW1 2BU, UK: daniellereddi@gmail.com

Received 24 June 2013 Revised 10 December 2013 Accepted 22 January 2014 Published Online First 26 February 2014
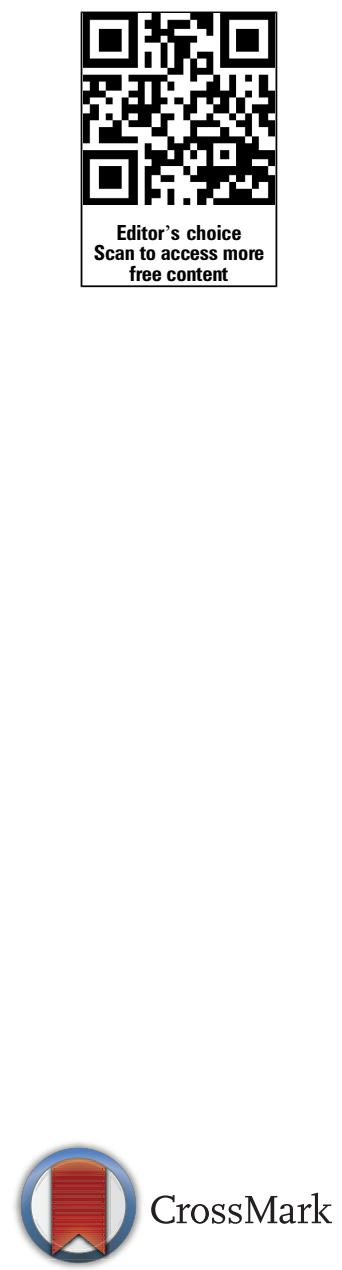

To cite: Reddi D, Curran N. Postgrad Med J

2014;90:222-227.

\section{ABSTRACT}

Interest in chronic pain after surgery has grown since the finding that more than a fifth of patients attending chronic pain clinics cite surgery as the cause for their chronic pain. The problem is not limited to major surgery; even common minor procedures such as hernia repair have a significant risk of chronic pain. Surgical technique can influence the development of chronic postsurgical pain (CPSP) and techniques to minimise nerve injury should be used where possible. Central nervous system changes contribute to the development of persistent pain following surgical trauma and nerve injury. Pharmacological agents that interrupt the mechanisms contributing to central sensitisation may be helpful in reducing the incidence of CPSP. Psychosocial factors are also important in the development of chronic pain and should be addressed as part of a holistic approach to perioperative care.

\section{INTRODUCTION}

Surgery is recognised as one of the most frequent causes of chronic pain in patients attending pain clinics. A survey of over 5000 patients found that the largest group, $34.2 \%$, had pain from degenerative disease, but the second largest group, $22.5 \%$, had developed chronic pain following surgery. ${ }^{1}$ Usually defined as pain persisting for more than 3 months after surgery, chronic pain is a recognised complication of many common procedures (table 1) and is the most serious long-term complication following inguinal hernia repair. ${ }^{2}$ Chronic pain has a significant impact on the quality of life and daily activities of sufferers. There is also a significant cost to society in terms of subsequent healthcare costs and social support systems. ${ }^{3}$

Table 1 Approximate numbers of operations carried out in England and incidence of chronic postsurgical pain (adapted from Macrae $2008^{4}$ )

\begin{tabular}{llc}
\hline $\begin{array}{l}\text { Type of } \\
\text { operation }\end{array}$ & $\begin{array}{l}\text { Incidence of } \\
\text { chronic pain (\%) }\end{array}$ & $\begin{array}{l}\text { No. of operations } \\
\text { in UK 2005-2006 }\end{array}$ \\
\hline $\begin{array}{l}\text { Total operations } \\
\text { Mastectomy }\end{array}$ & $20-50$ & 7125000 \\
$\begin{array}{l}\text { Caesarean } \\
\text { section }\end{array}$ & 6 & 18000 \\
Amputation & $50-85$ & 139000 \\
Cardiac surgery & $30-55$ & 15000 \\
Hernia repair & $5-35$ & 29000 \\
Cholecystectomy & $5-50$ & 75000 \\
Hip replacement & 12 & 51000 \\
Thoracotomy & $5-65$ & 61000 \\
\hline
\end{tabular}

This paper will explore the pathophysiological mechanisms that contribute to chronic postsurgical pain (CPSP), and the surgical and psychological risk factors that have been identified. Surgical and pharmacological strategies to reduce the development of CPSP will also be discussed. We will also consider how psychosocial factors influence the development and maintenance of chronic pain and the relevance of this to CPSP.

\section{PATHOPHYSIOLOGY}

The trauma and inflammation that occurs from cutting and handling tissues during surgery activates nociceptors. Nociceptive stimuli are transduced into electrical impulses that are carried to the spinal cord via primary afferent A $\delta$ and C fibres. Primary afferent neurones synapse with secondary afferent neurones in the dorsal horn of the spinal cord and carry impulses to higher centres via the contralateral spinothalamic and spinoreticular pathways, the two main ascending pain pathways. There are multiple further projections to the cerebral cortex and other higher centres. Central processing of impulses leads to the experience of pain. The complex pathways of nociceptive transmission are well described ${ }^{5}$ (figure 1).

Inflammatory pain occurs because sensitising, inflammatory mediators including cytokines, bradykinin and prostaglandins are released from injured and inflammatory cells at the site of tissue damage. Nociceptors demonstrate reversible plasticity in response to inflammatory mediators. The activation threshold of nociceptors is lowered, resulting in enhanced pain sensitivity at the site of tissue injury (peripheral sensitisation). ${ }^{6}$ Non-steroidal antiinflammatory drugs inhibit the production of prostaglandin E2 via locally induced cyclooxygenase- 2 enzymes and hence reduce peripheral sensitisation and pain. ${ }^{6}$ This type of inflammatory pain, secondary to local excitability, usually subsides once the source of the mediators subsides, as tissue healing occurs or the disease process is controlled. Heightened pain sensitivity can contribute to healing by helping to protect the damaged body part until repair has occurred. ${ }^{6}$

The central nervous system also demonstrates plasticity in response to pain, and pain signalling within the spinal cord can be enhanced. With ongoing nociceptive input, the stimulus-response relationship is altered and an increase in excitability of neurones in the central nervous system may occur, known as central sensitisation. Clinically this manifests as an increased response to painful stimuli (hyperalgesia), and pain secondary to normally non-painful tactile stimuli (allodynia). ${ }^{9}$ 
Figure 1 Ascending pain pathways ${ }^{8}$ reproduced with permission. DRG, dorsal root ganglion; PAG, periaqueductal grey matter.

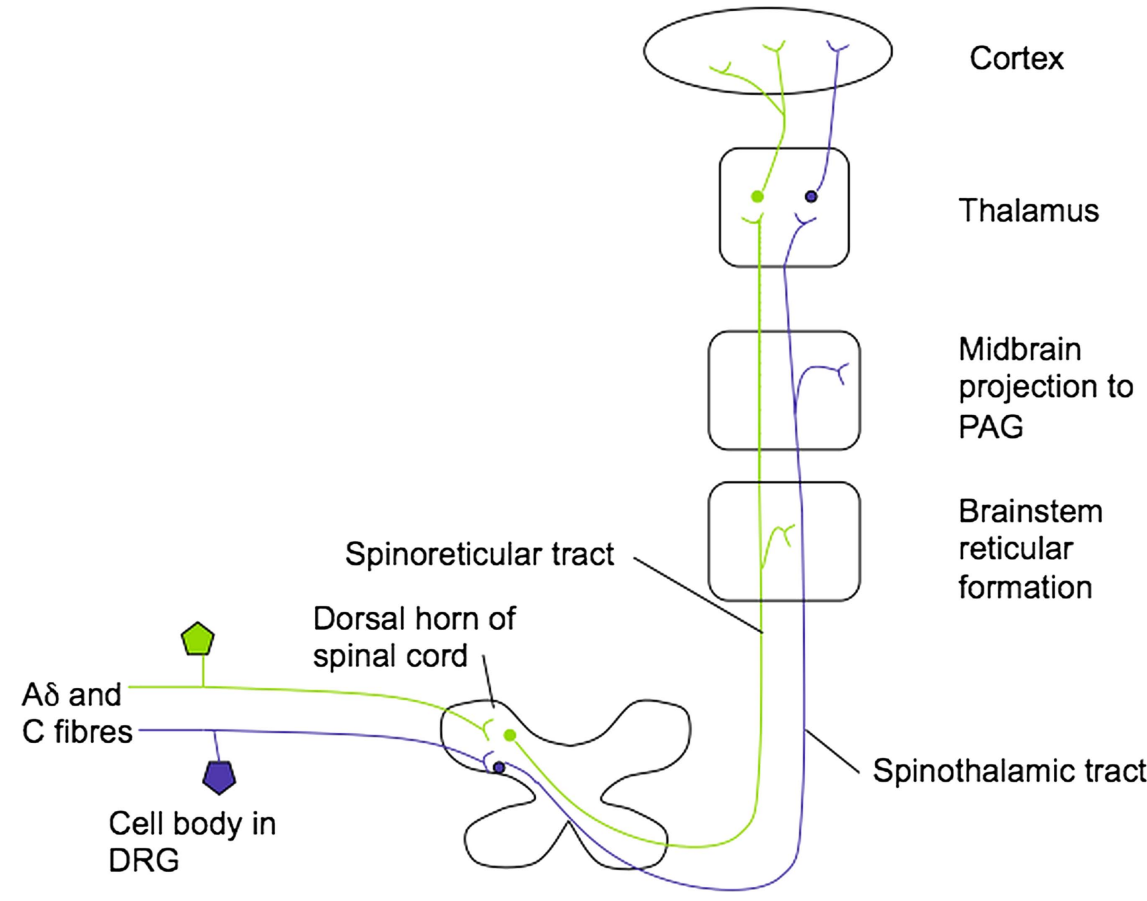

Wind-up, long-term potentiation and secondary hyperalgesia are all processes associated with central sensitisation. Wind-up occurs with repeated activation of $\mathrm{C}$ fibres and is due to the action of glutamate at NMDA (N-methyl-D-aspartate) receptors. Under normal conditions, a magnesium ion blocks the NMDA receptor. With ongoing painful stimuli, the magnesium block is removed and the response of second-order neurones to painful stimuli is amplified. This explains why NMDA receptor antagonists, such as ketamine, are useful in attenuating or blocking wind-up. ${ }^{10}$ The response of second-order neurones may outlast the initial stimulus and this is known as long-term potentiation, contributing to hyperalgesia. A lowering of the pain threshold outside of the area of inflammation (secondary hyperalgesia) occurs because of increased activation of second-order neurones in the dorsal horn of the spinal cord.

Nerve damage plays a role in CPSP. Following nerve injury, spontaneous ectopic discharges from injured nerves and nearby

Table 2 Risk factors for chronic postsurgical pain (reproduced with permission ${ }^{5}$ and adapted from ${ }^{7}$ and $^{4}$ )

\begin{tabular}{ll}
\hline Preoperative factors & Pain, moderate to severe, lasting more than 1 month \\
& Repeat surgery \\
& Psychological vulnerability (eg, catastrophising) \\
& Preoperative anxiety \\
& Female gender \\
& Younger age (adults) \\
& Workers' compensation \\
& Genetic predisposition \\
& Inefficient diffuse noxious inhibitory control (DNIC)-a \\
& descending \\
& pathway of pain inhibition \\
Intraoperative & Surgical approach with risk of nerve damage \\
factors & Pain (acute, moderate to severe) \\
Postoperative & Radiation therapy to area \\
factors & Neurotoxic chemotherapy \\
& Depression \\
& Psychological vulnerability \\
& Neuroticism \\
& Anxiety \\
\hline
\end{tabular}

uninjured nerves lead to spontaneous pain. ${ }^{11}$ The increased nociceptive input in to the dorsal horn contributes to central sensitisation. ${ }^{12}$ A loss of inhibitory interneurons in the dorsal horn results in a disinhibition of pain pathways and facilitation of pain transmission. ${ }^{13}$

The process of central sensitisation is thought to be important for the development of persistent pain; ${ }^{14}$ hence, surgical techniques and pharmacological interventions to minimise central sensitisation are of great interest.

\section{RISK FACTORS}

Risk factors have been identified for the development of CPSP (table 2). Some of these, such as genetic predisposition and gender, are not modifiable; however, it may be possible to reduce the risk by giving consideration to surgical approach, pain management and psychological predisposition.

\section{SURGICAL RISK FACTORS AND PREVENTION}

Surgical technique is a recognised risk factor in CPSP, ${ }^{15}{ }^{16}$ and it is likely that intraoperative nerve injury plays a role. ${ }^{7}$ Many of the operations associated with the development of chronic pain involve major nerves in the surgical field, for example, breast (intercostobrachial nerve) and thoracic surgery (intercostal nerves). Intraoperative nerve injury should be minimised to reduce the development of chronic pain. ${ }^{7} 17$ Laparoscopic surgery may reduce the risk of intraoperative nerve damage. For example, there is evidence for a lower incidence of persistent pain following laparoscopic hernia repair. ${ }^{2}$ Women who undergo breast cancer surgery in a high-volume unit by experienced surgeons are also less likely to experience chronic pain, and this is thought to be due to surgical technique and less nerve damage. ${ }^{16}$

Many operations do not involve transection of nerve trunks, but nerve injury may occur by stretching or crushing of nerves during tissue retraction or in cutting skin, viscera, fascia, muscles and joints, all of which are innervated by sensory nerves. ${ }^{17}$ However, it is apparent that factors other than nerve injury are an important cause of chronic pain. Intraoperative nerve transection does not always result in neuropathic pain, 
and avoiding nerve transection does not necessarily prevent chronic pain. ${ }^{4}$ The duration of surgery influences the development of chronic pain, with operations lasting greater than $3 \mathrm{~h}$ associated with an increased risk. ${ }^{18}$ This may be a reflection of complex pathology and the degree of intraoperative tissue damage.

While surgery is generally indicated or appropriate, there are situations where it is unnecessary and potentially unhelpful. The possibility of developing chronic pain after surgery should be considered with the indications for surgery. Chronic pain is a recognised problem after cosmetic breast augmentation, and patients should be fully informed of the risk. ${ }^{19}$ Careful surgical consideration should be given to patients with abdominal pain and visceral hyperalgesia syndromes, with no cause for pain found despite multiple investigations. Surgeons may feel pressure to investigate surgically, which can lead to a cycle of repeat laparoscopies and laparotomies, and worsening pain. ${ }^{4}$

\section{REGIONAL ANAESTHETIC AND ANALGESIC CONSIDERATIONS}

Considering the pathophysiological mechanisms proposed to contribute to chronic pain, it would seem logical that good perioperative pain control should have a long-term benefit by reducing central sensitisation.

\section{Regional anaesthesia}

Regional anaesthetic techniques use local anaesthetics to block the conduction of impulses along nerves. By minimising the transmission of signals to or within the spinal cord, it may be possible to prevent the barrage of nociceptive inputs into the dorsal horn and hence prevent central sensitisation. Spinal and epidural techniques act on the nerve roots while peripheral nerve blocks, plexus blocks and wound infiltration interrupt impulses in peripheral nerves. A recent Cochrane review found epidural analgesia for thoracotomy to be beneficial in reducing the risk of chronic pain at 6 months, and for paravertebral blocks in reducing chronic pain following breast cancer surgery at 5-6 months. ${ }^{20}$ Spinal anaesthesia has also been shown to be beneficial relative to general anaesthesia in reducing the risk of chronic pain after caesarean section. ${ }^{21}$ Limb amputation is associated with a high risk of chronic pain. Nerve injury is unavoidable; however, perioperative epidural analgesia reduces the incidence of severe phantom limb pain. ${ }^{5}$ Although there is some evidence for the benefit of regional anaesthetic techniques in reducing persistent pain after surgery, it is not clear-cut for all procedures.

\section{Pre-emptive and preventive analgesia}

The optimal timing and mode of perioperative regional and systemic analgesia is much debated. Pre-emptive analgesia is delivered prior to skin incision. Pre-emptive epidural analgesia has a significant benefit on postoperative pain $;^{5}$ however, the benefit other types of analgesia provided pre-emptively is not so clear. $^{22}$

Preventive analgesia describes an analgesic treatment that outlasts its expected duration. It may be important in minimising chronic pain after surgery through a reduction in central sensitisation. It is thought that for an analgesic regimen to be effective in preventing sensitisation of the nervous system it should act over the period of time during which there is an inflammatory stimulus, not just at skin incision, but into the postoperative period. $^{22}$ There is level I evidence that perioperative NMDA receptor antagonists show preventive analgesic affects, and this is thought to be due to their action in preventing central sensitisation. ${ }^{10}$

\section{Ketamine}

Ketamine is an NMDA receptor antagonist, and it is via this mechanism that it prevents NMDA receptor-mediated wind-up. It also acts as an analgesic via activation of descending inhibitory monoaminergic pain pathways and prevents opioid-induced hyperalgesia (a paradoxical increased sensitivity to painful stimuli in a patient receiving opioids) and acute opioid tolerance. Ketamine is rarely used as sole anaesthetic due to psychotomimetic adverse effects, but it can be a very useful adjuvant drug in patients requiring high levels of opioids. It potentiates opioid analgesia, improves pain control and reduces opioid-related side effects. ${ }^{23}$ It has been administered as a single low-dose bolus $(0.15-0.5 \mathrm{mg} / \mathrm{kg})$ or low-dose bolus followed by low-dose infusion ( 2 to $4 \mathrm{mcg} / \mathrm{kg} / \mathrm{min}$ ) in opioid-naïve patients undergoing a variety of surgeries. Most studies show a reduction in postoperative opioid requirements and pain that is most prominent in the first $24 \mathrm{~h},{ }^{24}$ although a long-term benefit has been shown in some studies. ${ }^{25}$ Perioperative epidural analgesia combined with intravenous ketamine reduces hyperalgesia and chronic pain after colonic surgery compared with intravenous analgesia alone. $^{26}$

\section{Gabapentinoids}

Gabapentin was initially introduced as an anticonvulsant in the 1990s. Subsequent trials showed it to be beneficial in the treatment of neuropathic pain. ${ }^{27}$ It acts at the $\alpha-2-\delta$ subunit of presynaptic calcium channels, inhibiting calcium influx and subsequent release of excitatory neurotransmitters in the pain pathways. ${ }^{28}$ Pregabalin is structurally similar to gabapentin. It has greater analgesic potency and a more favourable pharmacokinetic profile relative to gabapentin.

There is evidence from randomised controlled trials supporting the use of pre-emptive gabapentin in reducing acute postoperative pain. ${ }^{29} 30$ A systematic review and meta-analysis on the development of CPSP after perioperative gabapentin and pregabalin has recently been published. A positive effect of gabapentin was found in four out of eight studies. Among the six gabapentin trials that could be included in the meta-analysis, gabapentin caused a moderate-to-large reduction in the development of CPSP. ${ }^{31}$ All three studies included in the systematic review of the effects of pregabalin on CPSP showed significant preventive analgesic effects. Four of the six studies that analysed long-term functional outcomes found benefits of gabapentin and pregabalin. This analysis provides evidence that the reduction in CPSP may be linked to perioperative administration of these medications. However, there were a limited number of studies in this review, and this, along with the suggestion that publication bias may be present, means that future well-designed studies are needed to clarify whether gabapentin and pregabalin have a perioperative role in the prevention of CPSP.

\section{Intravenous lignocaine}

A meta-analysis of intraoperative intravenous lignocaine found a reduction in acute postoperative pain, with those undergoing abdominal surgery demonstrating the greatest benefit. ${ }^{32}$ Systemic local anaesthetics have also been found to have antihyperalgesic effects that last for days after surgery, long after the local anaesthetic action of the drug. The mechanism of action is not fully understood and cannot be explained by blockade of sodium channels. ${ }^{33}$ However, these findings suggest that lignocaine may have a role in preventing central sensitisation and hence as a preventive analgesic. 


\section{PSYCHOSOCIAL FACTORS}

The experience of pain is more than the detection of noxious stimuli transmitted to the central nervous system. Pain perception is influenced by mood, memories, expectation and social environment and so psychosocial factors should be considered alongside pain assessment and management.

The fear-avoidance model, thought to be important in the initiation and maintenance of chronic pain, demonstrates the importance of psychological factors in chronic pain (figure 2). In this model, a person believes that pain signals danger or harm. Fear of pain leads to avoidance of activities that may induce or exacerbate pain. Fear-avoidance behaviour may ultimately lead to disuse and disability, ${ }^{34}$ and a fear of pain has actually been found to better predict disability than the pain itself. ${ }^{35}$ Individuals who 'catastrophise' are more likely to enter into this cycle of fear-avoidance behaviour. Catastrophising can be described as an almost immediate habitual negative appraisal of a situation. ${ }^{36} \mathrm{~A}$ person might believe, for example, that if they bend their back then it will break and they will become wheelchair users. The relationship between the experience of pain and behavioural activity is mediated by expectation of pain and the anticipation of catastrophic consequences if certain activities are carried out. ${ }^{37}$ Anticipation of pain should be a helpful, adaptive response leading to modification of behaviour to avoid pain. However, it can become maladaptive in the chronic pain setting. Neuroimaging studies examining how anticipation and anxiety can heighten pain perception have revealed certain regions to be important in amplifying the pain experience. These regions include the entorhinal complex, amygdalae, anterior insular and prefrontal cortices. ${ }^{38}$

Psychological factors play a significant role in both acute and chronic back pain, and in the transition from acute to chronic pain. Psychosocial factors have in fact been found to have a greater impact than biomedical or biomechanical factors on back pain disability. ${ }^{40}$ Psychological factors are also known to influence acute postoperative pain, for example, Katz et al found a correlation between preoperative anxiety and acute postoperative pain in women undergoing breast cancer surgery. ${ }^{41}$ There is less in the literature concerning the role of psychosocial factors in pain persisting after surgery, although psychological vulnerability (eg, catastrophising), anxiety, depression, neuroticism and late return to work have all been found to be risk factors. ${ }^{5}{ }^{42}$ Patients with a fear of the long-term consequences of surgery are also at increased risk of long-term pain and poor recovery. ${ }^{43}$
Making sense of pain, what it may mean and what it does not mean are essential for effective coping. ${ }^{36}$ Pre-emptive cognitive and behavioural interventions decrease anxiety before and after surgery, reduce postoperative pain intensity and analgesic use. ${ }^{44}$ A study by Egbert et al nearly 50 years ago demonstrated a reduction in morphine requirements and length of stay when patients were provided with preoperative information about likely postoperative treatments and discomfort and instruction on relaxation techniques. ${ }^{45}$ When patients are given information about what they should expect to feel, in addition to procedural information, they experience less pain and distress compared with either type of information given alone. ${ }^{46}$ Relating this back to the fear-avoidance model we can see that information can provide reassurance that the sensations experienced after a procedure are normal and non-threatening. For example, following a total knee replacement, patients are encouraged to mobilise within hours of surgery. This may be uncomfortable, but it is important that patients are reassured that pain does not equal damage, that it is normal to experience some discomfort and it does not mean that they are jeopardising their operation or that something is wrong.

Educating patients and their carers about pain encourages a more positive attitude to pain relief $f^{5}$ and where possible we should try to involve patients in their pain management strategy. ${ }^{36}$ Through active involvement patients develop a greater sense of self-efficacy regarding treatment and disease-related behaviours and may be more likely to follow through on management decisions reached. ${ }^{47}$ Patients who respond passively to pain show greater distress and disability compared with those who attempt to solve the problem. Taking some control over the cause of pain or the method of analgesia has a beneficial effect. ${ }^{36}$

Social relationships can also influence pain reporting and disability. A study of chronic back pain patients found that when a partner is present who is attentive and likely to help them to avoid activities that may exacerbate pain then a patient is likely to report more pain and underperform on tasks. The expressions of pain are reinforced by the partner who acts to alleviate the patient's behavioural activity, and this is reflected in the increased level of pain. ${ }^{37}$

Managing expectation is recognised as an important component of enhanced recovery programmes. Carefully presented information from surgeons, anaesthetists and nurses about the surgical procedure, anticipated sensory experiences, analgesic
Figure 2 The fear-avoidance model (figure reproduced with permission of International Association for the Study of Pain $^{39}$ ).

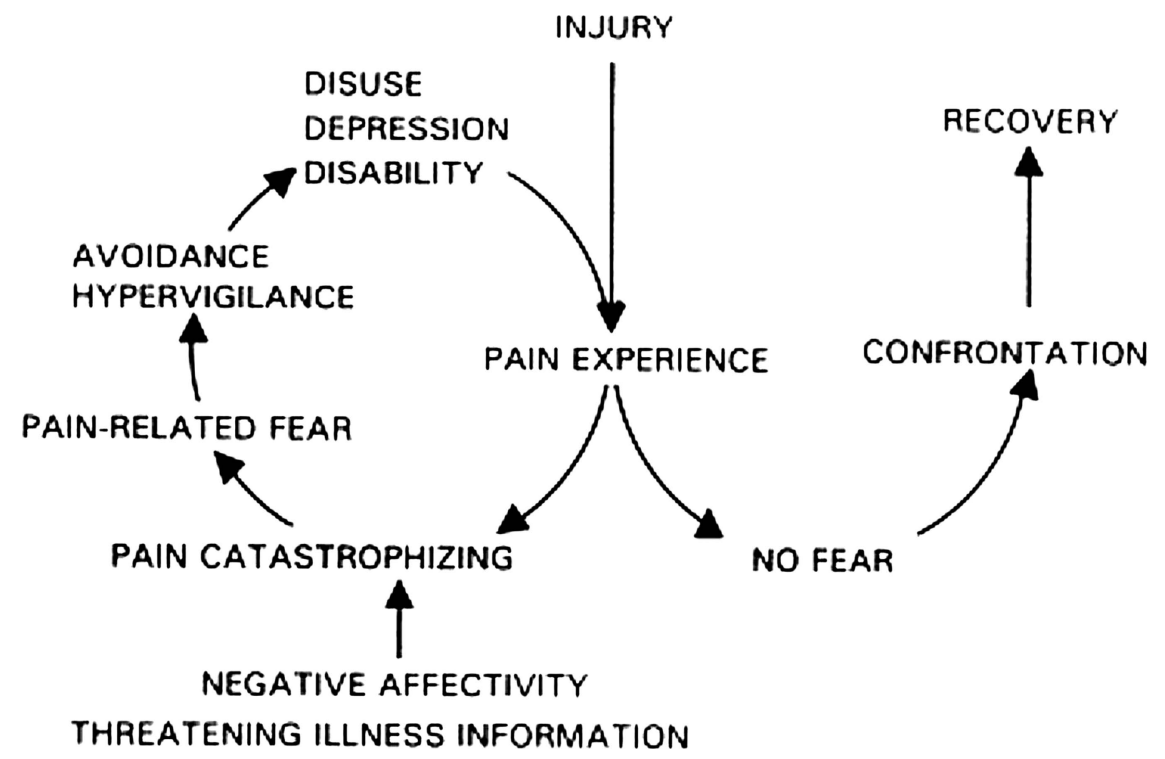


treatment and recovery period is expected to reduce anxiety and promote recovery. ${ }^{48}$ By addressing these issues in the perioperative period, it may be that we can also reduce the progression to persistent pain and disability in those at risk.

\section{CONCLUSION}

By understanding the physiological processes that contribute to persistent pain, we can use therapeutic interventions to minimise the progression from acute to chronic pain. An awareness of the risk factors for CPSP may help to identify those at high risk and target interventions accordingly. The impact of psychological factors on the pain experience should always be considered, and a collaborative approach involving patients in their treatment decisions is recommended for patients to experience the best outcome.

\section{Main messages}

- Chronic postsurgical pain makes a significant contribution to the burden of chronic pain.

- Perioperative pain contributes to central sensitisation and subsequent chronic postsurgical pain.

- Surgical technique and intraoperative nerve injury are risk factors in chronic postsurgical pain.

- Regional anaesthetic techniques reduce the risk of chronic postsurgical pain following breast cancer surgery and thoracotomy.

- Adjuvant analgesic drugs including ketamine, gabapentin and pregabalin may be useful as preventive analgesics, reducing chronic postsurgical pain.

\section{Current research questions}

What are the genetic factors that predispose individuals to chronic postsurgical pain?

- Why do some patients with intraoperative nerve damage develop chronic postsurgical pain whereas others do not?

- Is it possible to identify patients with psychological risk factors for chronic postsurgical pain preoperatively and modify their risk?

\section{Key references}

- Macrae WA. Chronic post-surgical pain: 10 years on. Br J Anaesth 2008;101: 77-86.

- Macinytre PE, Schug SA, Scott DA, et al.; APM:SE Working Group of the Australian and New Zealand College of Anaesthetists and Faculty of Pain Medicine. Acute Pain Management: Scientific Evidence. 3rd ed. Melbourne: ANZCA \& FPM, 2010.

- Eccleston C. Role of psychology in pain management. $\mathrm{Br} J$ Anaesth 2001;87(1):144-52.

- Vlaeyen JW, Linton SJ. Fear-avoidance and its consequences in chronic musculoskeletal pain: a state of the art. Pain 2000;85 : 317-32.

- Kehlet H, Jensen TS, Woolf CJ. Persistent postsurgical pain: risk factors and prevention. Lancet 2006;367 : 1618-25.

\section{Self assessment questions}

Please answer true or false for the below statements,

1. Moderate-to-severe postoperative pain is a risk factor for chronic postsurgical pain.

2. Perioperative epidural analgesia has no impact on chronic postsurgical pain.

3. Perioperative ketamine has no effect on postoperative opioid requirements or side effects.

4. Gabapentin may be useful in preventing chronic postsurgical pain.

5. Surgical technique can influence chronic postsurgical pain.

Competing interests None.

Provenance and peer review Not commissioned; externally peer reviewed.

\section{REFERENCES}

1 Crombie IK, Davies HTO, Macrae WA. Cut and thrust: antecedent surgery and trauma among patients attending a chronic pain clinic. Pain 1998;76:167-71.

2 Jenkins JT, O'Dwyer PJ. Inguinal hernias. BMJ 2008;336:269-72.

3 Turk DC. Clinical effectiveness and cost-effectiveness of treatments for patients with chronic pain. Clin J Pain 2002;18:355-65.

4 Macrae WA. Chronic post-surgical pain: 10 years on. Br J Anaesth 2008:101:77-86.

5 Macinytre PE, Schug SA, Scott DA, et al.; APM:SE Working Group of the Australian and New Zealand College of Anaesthetists and Faculty of Pain Medicine. Acute Pain Management: Scientific Evidence. 3rd ed. Melbourne: ANZCA \& FPM, 2010.

6 Woolf CJ, Ma Q. Nociceptors-noxious stimulus detectors. Neuron 2007;55:353-64.

7 Kehlet $\mathrm{H}$, Jensen TS, Woolf CJ. Persistent postsurgical pain: risk factors and prevention. Lancet 2006:367:1618-25.

8 Reddi D, Curran N, Stephens R. An introduction to pain pathways and mechanisms. Br J Hosp Med 2013;74:C188-91.

9 D'Mello R, Dickenson AH. Spinal cord mechanisms of pain. Br J Anaesth 2008; 101:8-16.

10 McCartney CJL, Sinha A, Katz J. A qualitative systematic review of the role of $\mathrm{N}$-Methyl-D-aspartate receptor antagonists in preventive analgesia. Anesth Analg 2004:98:1385-400.

11 Baron R, Binder A, Wasner G. Neuropathic pain: diagnosis, pathophysiological mechanisms, and treatment. Lancet Neurol 2010:9:807-19.

12 Woolf CJ, Mannion RJ. Neuropathic pain : aetiology, symptoms, mechanisms, and management. Lancet 1999;353:1959-64.

13 Woolf CJ, Salter MW. Neuronal Plasticity: Increasing the Gain in Pain. Science 2000:288:1765-8.

14 Sandkuhler J. Models and Mechanisms of Hyperalgesia and Allodynia. Physiol Rev Supp/ 2009;89:707-58.

15 Maguire MF, Ravenscroft A, Beggs D, et al. A questionnaire study investigating the prevalence of the neuropathic component of chronic pain after thoracic surgery. Eur J Cardiothoracic Surg 2006;29:800-5.

16 Tasmuth T, Blomqvist C, Kalso E. Chronic post-treatment symptoms in patients with breast cancer operated in different surgical units. Eur J Surg Oncol 1999:25:38-43.

17 Katz J, Seltzer Z. Transition from acute to chronic postsurgical pain: risk factors and protective factors. Expert Rev Neurother 2009;9:723-44.

18 Peters ML, Sommer M, de Rijke JM, et al. Somatic and psychologic predictors of long-term unfavorable outcome after surgical intervention. Ann Surg 2007:245:487-94.

19 von Sperling ML, Høimyr H, Finnerup K, et al. Persistent pain and sensory changes following cosmetic breast augmentation. Eur J Pain 2011;15:328-32.

20 Andreae $\mathrm{MH}$, Andreae DA. Local anaesthetics and regional anaesthesia for preventing chronic pain after surgery. Cochrane Database of Systematic Reviews 2012;(10).

21 Nikolajsen L, Sørensen HC, Jensen TS, et al. Chronic pain following Caesarean section. Acta anaesthesio/ Scand 2004:48:111-16.

22 Kissin I. Preemptive Analgesia. Anesthesiology 2000;93:1138-43.

23 Hirota K, Lambert DG. Ketamine: new uses for an old drug? Br J Anaesth 2011;107:123-6.

24 Carroll I, Angst M, Clark J. Management of perioperative pain in patients chronically consuming opioids. Reg Anesth Pain Med 2004;29:576-91.

25 De Kock M, Lavand'homme P, Waterloos H. "Balanced analgesia" in the perioperative period: is there a place for ketamine? Pain 2001:92:373-80. 
26 Lavand'homme P, De Kock M, Waterloos H. Intraoperative epidural analgesia combined with ketamine provides effective preventive analgesia in patients undergoing major digestive surgery. Anesthesiology 2005;103:813-20.

27 Gilron I, Bailey JM, Tu D, et al. Morphine, Gabapentin, or Their Combination for Neuropathic Pain. N Engl J Med 2005:352:1324-34.

28 Rowbotham DJ. Gabapentin: a new drug for postoperative pain? $\mathrm{Br} J$ Anaesth 2006:96:152-5.

29 Dirks J, Fredensborg BB, Christensen D, et al. A Randomized Study of the Effects of Single-dose Gabapentin versus Placebo on Postoperative Pain and Morphine Consumption after Mastectomy. Anesthesiology 2002;97:560-4.

30 Pandey CK, Navkar DV, Giri PJ, et al. Evaluation of the Optimal Preemptive Dose of Gabapentin for Postoperative Pain Relief After Lumbar Discectomy. J Neurosurg Anesthesiol 2005;17:65-8

31 Clarke $\mathrm{H}$, Bonin RP, Orser B, et al. The prevention of chronic postsurgical pain using gabapentin and pregabalin: a combined systematic review and meta-analysis. Anesth Analg 2012:115:428-4.

32 Vigneault L, Turgeon AF, Côté $D$, et al. Perioperative intravenous lidocaine infusion for postoperative pain control: a meta-analysis of randomized controlled trials. Can J Anaesth 2011:58:22-37.

33 Strichartz GR. Novel ideas of local anaesthetic actions on various ion channels to ameliorate postoperative pain. Br J Anaesth 2008;101:45-7.

34 Leeuw M, Goossens MEJB, Linton SJ, et al. The fear-avoidance model of musculoskeletal pain: current state of scientific evidence. J Behav Med 2007;30:77-94.

35 Crombez G, Vlaeyen JW, Heuts PH, et al. Pain-related fear is more disabling than pain itself: evidence on the role of pain-related fear in chronic back pain disability. Pain 1999:80:329-39.

36 Eccleston C. Role of psychology in pain management. Br J Anaesth 2001;87:144-52.

37 Morley S. Psychology of pain. Br J Anaesth 2008;101:25-31.

38 Tracey I. Imaging pain. Br J Anaesth 2008;101:32-9.

39 Vlaeyen JW, Linton SJ. Fear-avoidance and its consequences in chronic musculoskeletal pain: a state of the art. Pain 2000;85:317-32.
40 Guzmán J, Esmail R, Karjalainen K, et al. Multidisciplinary rehabilitation for chronic low back pain: systematic review. BMJ 2001;322:1511-16.

41 Katz J, Poleshuck EL, Andrus CH, et al. Risk factors for acute pain and its persistence following breast cancer surgery. Pain 2005;119:16-25.

42 Hinrichs-Rocker A, Schulz K, Jänvinen I, et al. Psychosocial predictors and correlates for chronic post-surgical pain (CPSP)—A systematic review. Eur J Pain 2009;13:719-30.

43 Munafò MR, Stevenson J. Anxiety and surgical recovery. Reinterpreting the literature. J Psychosom Res 2001;51:589-96.

44 Carr DB, Goudas LC. Acute pain. Lancet 1999;353:2051-8.

45 Egbert LD, Battit GE, Welch CE, et al. Reduction of postoperative pain by encouragement and instruction of patients-a study of doctor-patient rapport. N Engl J Med 1964;270:825-7.

46 Suls J, Wan CK. Effects of sensory and procedural information on coping with stressful medical procedures and pain: a meta-analysis. J Consult Clin Psychol 1989;57:372-9.

47 Baars JE, Markus T, Kuipers EJ, et al. Patients' preferences regarding shared decision-making in the treatment of inflammatory bowel disease: results from a patient-empowerment study. Digestion 2010;81:113-19.

48 Kehlet $\mathrm{H}$, Dahl JB. Anaesthesia, surgery, and challenges in postoperative recovery. Lancet 2003;362:1921-8.

\section{Answers}

1. True

2. False

3. False

4. True

5. True 